\title{
A Performance Evaluation of Shape Based Image Retrieval Using Heuristic Function
}

\author{
Pushpatai Tejlal Tandekar ${ }^{1}$, Dhirendra Kumar $\mathrm{Jha}^{2}$ \\ p.tandekar@yahoo.in ${ }^{1}$, dhirendrajha@gmail.com ${ }^{2}$ \\ Department of Computer Science and Engineering ${ }^{1,2}$ \\ Malhotra Technical Research Institute, Bhopal, India ${ }^{1,2}$
}

\begin{abstract}
Heuristic function plays an important role in content based image retrieval. The heuristic function used for feature selection and feature optimization for retrieval process. The feature selection process are depends on feature extraction process. The content based image consists of three types of features such as color, texture and shape. The shape feature is very important feature for image retrieval. The extraction of shape feature various authors used different method such ad Gabor filter, wavelet transform function and Fourier descriptor. Now in current research trend MPEG-7 feature descriptor are mostly authors are used. In this paper descried the review of content based image retrieval based on shape based feature and optimization technique such as ANT colony optimization, genetic algorithm and neural network. The empirical evaluation result shows that ANT colony optimization technique is better optimization technique in compared with other such as genetic and neural network.
\end{abstract}

\section{Indexing terms/Keyword}

Image Retrieval, shape based feature extraction and heuristic function

\section{Council for Innovative Research}

\author{
Peer Review Research Publishing System
}

Journal: International Journal of Computers \& Technology

Vol 12, No 2

editor@cirworld.com

www.cirworld.com, member.cirworld.com 


\section{INTRODUCTION}

The image retrieval process is most important part of digital multimedia and satellite communication for better prediction of crops and weather. Large collections of images are becoming available to the community, from photo collection to web data, or even video databases. Since visual media requires large amounts of memory and computing power for processing and storage, there is a need to efficiently index and retrieve visual information from image database. A number of image features based on color and texture attributes have been developed[1,2]. A color histogram is a representation of the distribution of colors ratio mapping. For digital images, it is in essence the amount of pixels that have colors in each of a fixed list of color ranges that span the image color space, the set of all possible colors. Image content typically consists in low-level features directly extracted from images such as color, texture and shape[5]. Among these, shape represents one of the most significant features that are employed for image retrieval. The techniques, tools and algorithms that are used in CBIR, originate from many fields such as information, pattern recognition, signal processing, and computer visualization. It is a field of research that is attracting professionals from different industries like crime anticipation, drug structural design, and style and publishes. The various authors give a local attention, in CBIR; local features play a significant role in determining the similarity of images along with the shape information of the target[11,12]. Accurate segmentation technique is very difficult to achieve but is also not so critical in object shape resolve. A windowed search over location and scale is shown more effective in object based image retrieval than methods based on inaccurate segmentation. There is a diversity of techniques that has been proposed in the literature for shape feature extraction. Shape feature extraction techniques are divided into two categories: outer-based and inner-based. Outer based methods use only the contour or border of the object shape and completely ignore its internal. On the other hand, the area based techniques take into account internal details besides the outer details. Heuristic and Meta heuristic function play an important role in image retrieval process. The heuristic function used as feature selector and feature optimization. The diversity of heuristic function deals the semantic relation with query image and retrieval image[16]. The objective function of image retrieval system suggests the selection of heuristic function possibility. In the last years, the development of optimization algorithms have been inspired and influenced by natural and biological behaviors. Bio-inspired meta-heuristic optimization approaches provided new ways to achieve nearly-optimal solutions in highly nonlinear, multidimensional result area, with lower complexity and faster convergence than traditional algorithms.PSO is a population based metaheuristic optimization technique with stochastic nature, inspired by social behavior of bird flocking or search teaching. The system is initialized with a population of random solutions and searches for optima by update generation. The possible solutions, called particle, fly through the problem space by following the current optimum particles[20]. PSO has been successfully applied as an efficient optimization tool in image classification. This paper is divided into six sections. SectionI gives the introduction of image compression. Section-II gives the related of image retrieval with shape based. Feature extraction technique in section-III. In section IV discuss heuristic function. In section-V discuss experimental evaluation of all method and finally discuss conclusion and future work in section VI.

\section{RELATED WORK}

In this section described the related work about image retrieval based on shape feature extraction and optimization technique. The shape based technique is most important and recent approach for feature extraction process. In current scenario various methods are used for shape based feature extraction. The optimization of feature improves the retrieval capacity of image retrieval. Some work related discuss here.

[1] In this paper author proposed a method of fuzzy clustering is applied to shapes feature by Fourier descriptors in order to derive a set of prototypes representative of a set of shape categories. We apply a fuzzy clustering algorithm equipped with a partial supervised mechanism that takes advantage from domain knowledge expressed in terms of a number of labeled shapes. The derived prototypes are used as indexing mechanism to perform the retrieval process. Whenever a user submits a shape query, this is matched with all the prototypes and shapes belonging to categories corresponding to the most similar prototypes are provided as a result.

[2] In this paper authors proposed an automatic histological image classification system that uses biologically interpretable shape-base features. These features confine the distribution of shape pattern, described by the Fourier shape descriptors, in different strains of a histological image.

[3] In this paper authors give an for log-based relevance feedback that systematically integrates the log data of users' relevance judgments with regular relevance feedback for image retrieval. A user launches a query in a CBIR system for searching desired images in stored databases. Then, the CBIR system compute or match the similarity between the user query and the image samples in database using the low-level image features. Images among the high similarity determine are return to the user. Next, the user selects the relevance of the initially returned results and submits his or her judgments to the CBIR system. Relevance feedback approaches refine the early retrieval a result based on the user's relevance judgments, and returns an enhanced set of results to the user.

[4] In this paper authors proposed biased maximum margin analysis (BMMA) and a semi supervised BMMA for integrating the distinct properties of feedbacks and utilizing the information of unlabeled samples for SVM-based RF scheme. BMMA differentiate the positive feedbacks from negative ones based on general analysis; while the Semi-BMMA can effectively combine information of unlabeled samples by introduce a Laplacian regularize to the BMMA.

[5] In this paper authors proposed a novel approach for content based color image classification using Support Vector Machine. Traditional classification approaches deal poorly on content based image classification tasks being one of the reasons of high dimensionality of the feature space. Color image classification is done on features extracted from 
histograms of various color components. The advantage of using color image histograms is proved better efficiency, insensitivity to little change in camera view-point for translation and rotation etc.

[6] In this paper authors proposed novel fuzzy decision tree, the tree structure is determined via a class grouping algorithm, which define the sets of classes to be isolated from at each internal node, which is based on the degree of fuzzy confusion between the whole classes. Additionally the efficient feature selection is integrated within the tree construction process; select the suitable feature subsets necessary for the node discriminations separately. FDT-SVM exhibit several attractive merits such as an enhanced classification accuracy, interpretable hierarchy, and low form complexity. in addition, it provide hierarchical image segmentation and has reasonably low computational and data storage demands.

[7] in this paper authors used a process of the landscape of general strategies in visual content-based video indexing and retrieval, focusing on methods for video structure analysis, including shot boundary detection, key frame extraction and scene segmentation, extraction of features including static key frame features, object features and motion features, video data mining, video annotation, video retrieval including query interfaces, similarity measure and relevance feedback, and video browsing.

[8] In this paper authors proposed a method HDK that uses more than one clustering technique to improve the performance of CBIR. This method makes use of hierarchical and divide and conquer k-means clustering technique with equivalency and compatible relation concepts to improve the performance of the K-Means for using in high dimensional datasets.

[9] In this paper authors proposed a method of Shape-Based Image Retrieval Using Canny Edge Detection and K-Means Clustering Algorithms for numerous Medical Images, has been developed to choose the medical images from huge volume of medical image databases. Those require the preprocessing, for feature extraction and feature classification process, retrieval and indexing steps sequentially to choose an efficient medical image retrieval system. For preprocessing step, the image segmentation method has been carried out, for the feature selection, basic shape feature has been extracted using canny edge detection algorithm, and designed for classification, the K-means classification algorithm has been used. For retrieval of images, the Euclidian distance method values are calculated between query image and database images

[10] In this paper authors give a new framework for multimedia content analysis and retrieval which consists of two independent algorithms. The first part of propose a new semi-supervised algorithm called ranking with Local Regression and Global Alignment (LRGA) to learn a robust Laplacian matrix for data ranking. In the LRGA, at each data point, a neighborhood linear regression model is used to predict the ranking scores of its neighboring points. An integrated object function is then proposed for globally align the local models from all the data points so that an optimal ranking score can be assigned to each data point. Other is proposed a semi-supervised long-term Relevance Feedback (RF) algorithm to refine the multimedia data representation. The proposed continuing $\mathrm{RF}$ algorithms utilize the both multimedia data distribution in multimedia feature space and the history RF information provide by users. An outline ratio optimization difficulty is then formulated and solved by an efficient algorithm.

[11] In this paper authors used a 3-D object retrieval and recognition approach by exploring the higher order relationship among 3-D objects via hyper graphs. In this framework, multiple hyper graphs are constructed and the learning on the fused hyper graph is conducted for 3-D object retrieval and recognition.

[12] In this paper authors proposed a new algorithm called Priority Based Pheromone Algorithm belongs to Ant colony System to give better optimal solution for clustering. Concept of this algorithm is to build clusters using three potential values priority value, pheromone and heuristic information. This algorithm is compared with normal Ant colony algorithm, Genetic Algorithm and basic K-means algorithm. Comparison is done by percentage of errors, best fitness and maximum number of iterations.

\section{FEATURE EXTRACTION TECHNIQUE}

Feature extraction is the method of extracting the features from image to form a feature matrix. This feature matrix is then used by retrieval technique to retrieve the input unit with target output unit[14]. Here discuss some feature extraction technique. The hybrid shape descriptor ZFDR , containing both visual and geometric information of a 3D model, is composed of four parts: Zernike moments feature, Fourier descriptor feature, Depth information feature and Ray-based feature. The shape descriptor computation process consists of two steps: 3D model normalization and feature extraction. Continuous Principle Component Analysis (CPCA) alignment algorithm is utilized during the normalization step to align the 3D an image descriptor, which comprises Zernike moments and Fourier descriptors, is adopted to represent the features of a silhouette view. These two features characterize the visual information of a 3D model[15]. They are effective in representing certain types of models, while not as effective as depth buffer-based features for some other classes. Therefore, we devise a hybrid shape descriptor by also integrating certain geometric information of a 3D model. Particularly, we integrate the depth buffer-based feature and ray-based with spherical harmonic representation feature.

1) Cube-Based View Sampling: Considering the tradeoff between the feature extraction time and retrieval efficiency, the approach samples 13 silhouette views to represent a 3D model by setting cameras at the following locations on a cube: $(1,0,0),(0,1,0),(0,0,1),(1,1,1),(-1,1,1),(-1,-1,1),(1,-1,1),(1,0,-1),(0,1,-1),(1,1,0),(0,1,1),(1,0,1),(1,-1,0)$.

2) Zernike Moments Feature $(Z)$ : Zernike moments feature is utilized to extract the region-based features of a silhouette view. For each sample view, the algorithm computes the Zernike moments (up to the 10th order, totally 35 moments). 
Then, the Zernike moments features are concatenated orderly according to the order of the view sequence, resulting a 13_35 matrix as the Zernike moments feature of a 3D model.

3) Fourier Descriptor Feature $(F)$ : Centroid distance based Fourier descriptor is adopted to extract the contour feature of a silhouette view. The first 10 Fourier coefficients are used as the Fourier descriptor of a view. The combination of the Fourier descriptors of 13 views forms a 13_10 matrix as the Fourier descriptor feature of a 3D model.

4) Depth Information Feature (D): This feature is to extract the Fourier features of the six depth buffer images of a 3D model. First, the six depth views of a 3D model are rendered, then 2D Fourier Transform is applied on the depth views, and finally 438 Fourier coefficients are employed as the depth features of a 3D model.

5) Ray-Based Feature (R): First, a set of rays emanating from the center of the model are shoot and based on the outmost intersections between the rays and model, the ray-based feature vector in the spatial domain is extracted. Then, Spherical Harmonics Transform is applied on the obtained radial distance feature vector to transform it from the spatial domain to the spectral domain. Finally, a 136-dimensional feature vector is obtained to depict the ray based features of a 3D model.

6) Hybrid Shape Descriptor ZFDR Distance Computation: The hybrid shape descriptor ZFDR of a model is a combination of Zernike moments feature Z, Fourier descriptor F, Depth information feature D and Ray-based descriptor R. First, appropriate distance metrics are assigned to measure the component distances $\mathrm{dZ}, \mathrm{dF}, \mathrm{dD}$ and $\mathrm{dR}$ between two models, then the four component distances are linearly combined to form the hybrid descriptor distance $d$ ZFDR.

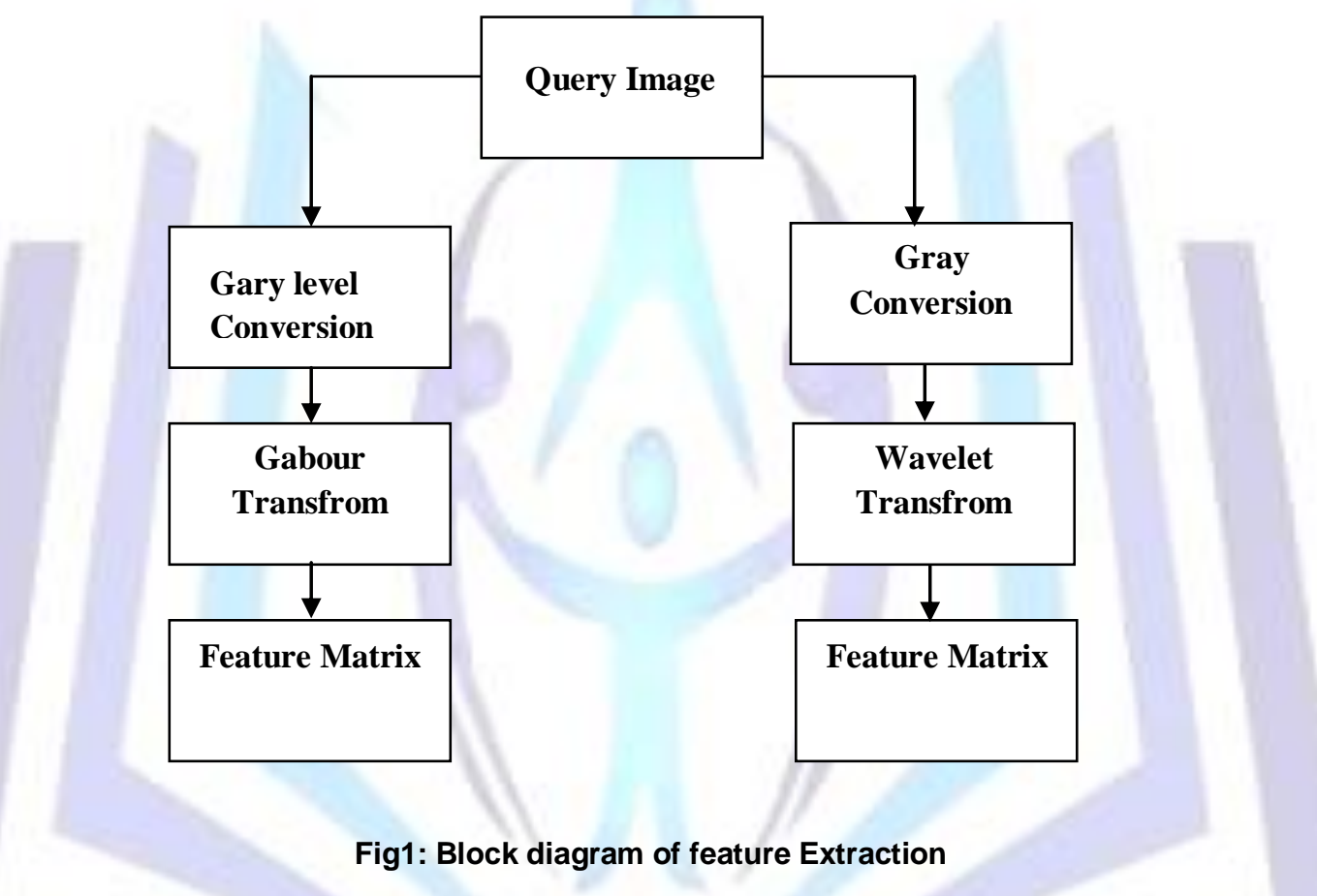

\section{HEURISTIC FUNCTION}

In this section heuristic function such as genetic algorithm, ant colony optimization and neural network. These heuristic function used as feature selector and feature optimizer in image retrieval process. The efficiency of content based retrieval varies according to the function of optimization. Some optimization algorithm discuss here. In this paper we used optimization process of feature optimization for image retrieval systems try to give better solutions for the selection of feature to current query image. The feature optimization is very significant because the feature is a main house block for making retrieval more efficient to user communities[18]. The optimization methods for Image retrieval are the main objective for this paper. The feature problem is just like NP-Complete. Therefore, approximation algorithms are careful, which are expected to quickly offer a solution, even if it is only near to-optimal. Image retrieval is a requirement for many Query image computing applications. Image retrieval might refer to the completion of allocated query, minimization of execution time for query. It might occur that these needs are satisfied only by specific feature, so that they only these feature can be assigned for that application [19]. Situations might become more complex when there are more query image having Image retrieval process, and several feature exist which satisfy them. Image retrieval constrains is another subject for the optimization process. In these cases, a number of challenging issues need to be addressed: maximization of system job completion and user satisfaction, the sites independence, and scalability. Performance prediction is also used in optimizing the feature extraction algorithms. Existing algorithms only consider an instant value of the performance at the feature, and assume this value remains constant during the task execution[17]. A more accurate model should consider that performance changes during the execution of the application. These Algorithms, usually find a solution close to the best one and they find it fast and easily. The ant colony optimization algorithm (ACO) is a heuristic algorithm for solving computational problems which can be reduced to finding good paths through graphs[19]. This algorithm is a member of ant colony algorithms family, in swarm intelligence methods. It is the first algorithm was aiming to search for an optimal path in a graph; based on the behavior of ants seeking a path between their colony and a source of food. The 
original idea has since diversified to solve a wider class of numerical problems, and as a result, several problems have emerged, drawing on various Aspects of the behavior of ants. Genetic algorithms are search algorithms based on the mechanics of natural selection and natural genetics. They combine survival of the fittest among string structures with a structured yet randomized information exchange to form a search algorithm with some innovative flair of human search. These algorithms are started with a set of random solution called initial population. Each member of this population is called a chromosome [20]. Each chromosome of this problem which consists of the string genes. The number of genes and their values in each chromosome depends on the population specification. In the algorithm of this paper, the number of genes of each chromosome is equal to the number of the nodes in the DGA and the gene values demonstrate the scheduling priority of the related task to the node, where the higher priority means that task must executed early.

\section{EXPERIMENTAL RESULT ANALYSIS}

To implement the above technique, we first find the shape features for images. The number of pixels in each edge is referred to as one feature. These features are then stored in a matrix in row by row fashion i.e. each row contains the shape features corresponding to one image. We evaluated performance of our algorithm using a general-purpose image database containing $500 \mathrm{JPEG}$ images with size of $256^{\star} 256$ or $256^{\star} 384$ pixels from COREL photo gallery. These images are divided into 5 categories, and there are 100 images in each semantic category. We test the performance of; the retrieval performance is measured by precision and recall, which are defined as

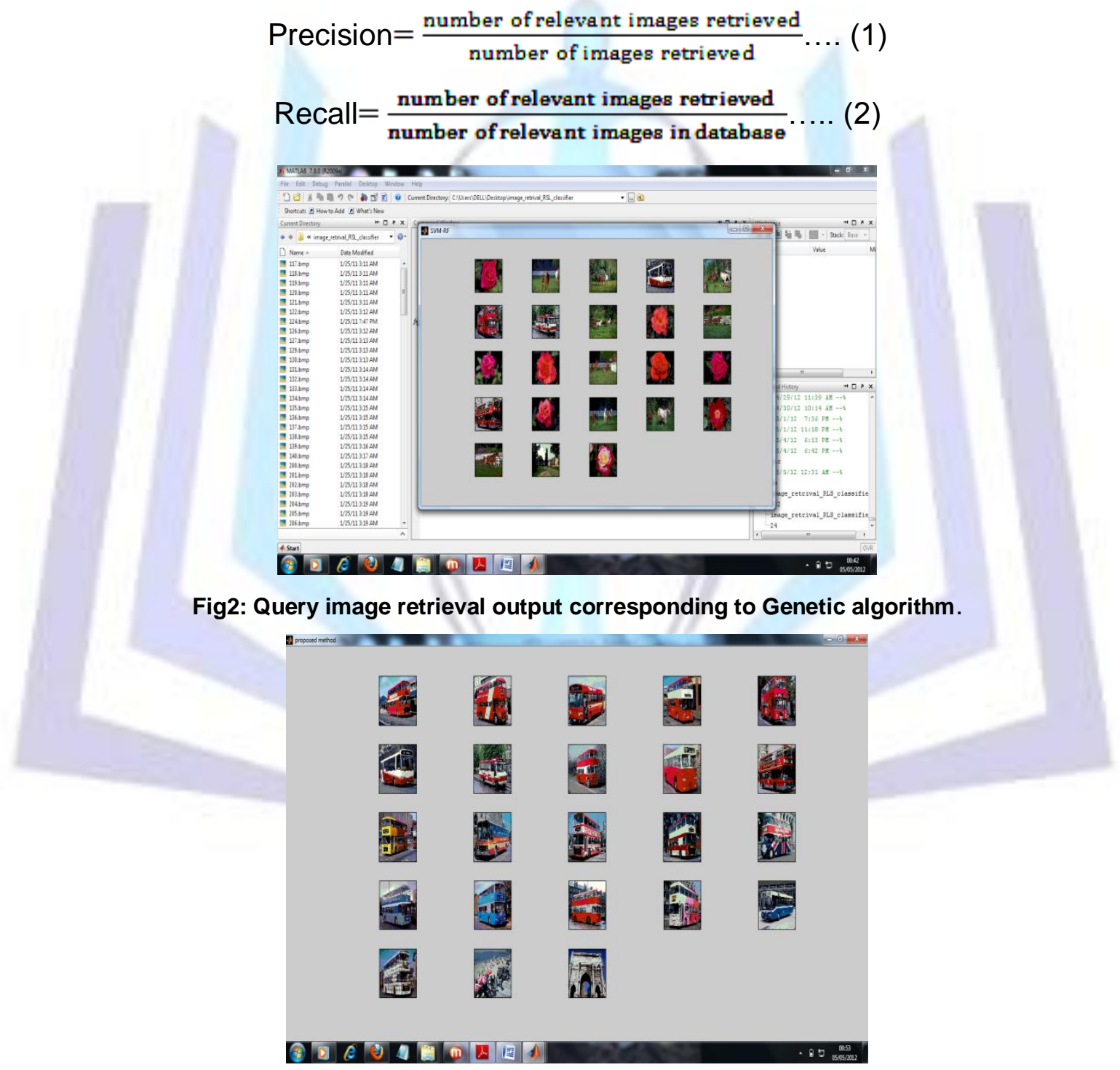

Fig 3: Query image retrieval output corresponding to Ant Colony Optimization. 


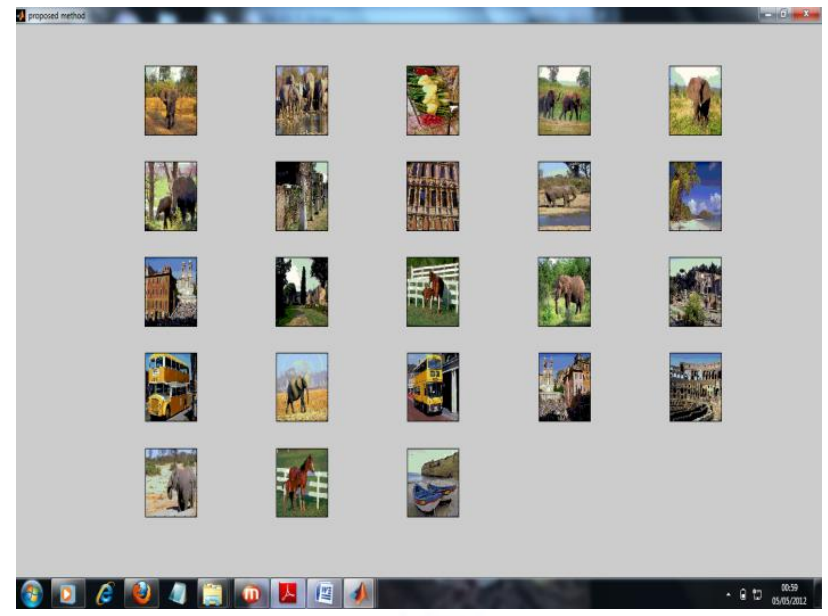

Fig 4: Query image retrieval output corresponding to Neural Network.

The result analysis of image classification based on number of class of image based on three methods. The total number of image in classification window is 500 and count the number of actual recall image in result windows

Table 1. Comparative Precision and Recall of image Retrieval

\begin{tabular}{|c|c|c|c|}
\hline Data set & Method & $\begin{array}{c}\text { Precision } \\
\text { (\%) }\end{array}$ & $\begin{array}{c}\text { Recall } \\
\text { (\%) }\end{array}$ \\
\hline \multirow{3}{*}{ Dataset-1 } & Gabor-GA & 88.66 & 82.22 \\
\cline { 2 - 4 } & Gabor-ANT & 92.33 & 85.00 \\
\cline { 2 - 4 } & Gabor-NN & 91 & 93 \\
\hline \multirow{3}{*}{ Dataset-2 } & Gabor-GA & 86.33 & 82.81 \\
\cline { 2 - 4 } & Gabor-ANT & 83 & 81 \\
\cline { 2 - 4 } & Gabor-NN & 85.33 & 78.83 \\
\hline
\end{tabular}

\section{Comarartive image retrival based on three herustic function for Gabor filter}

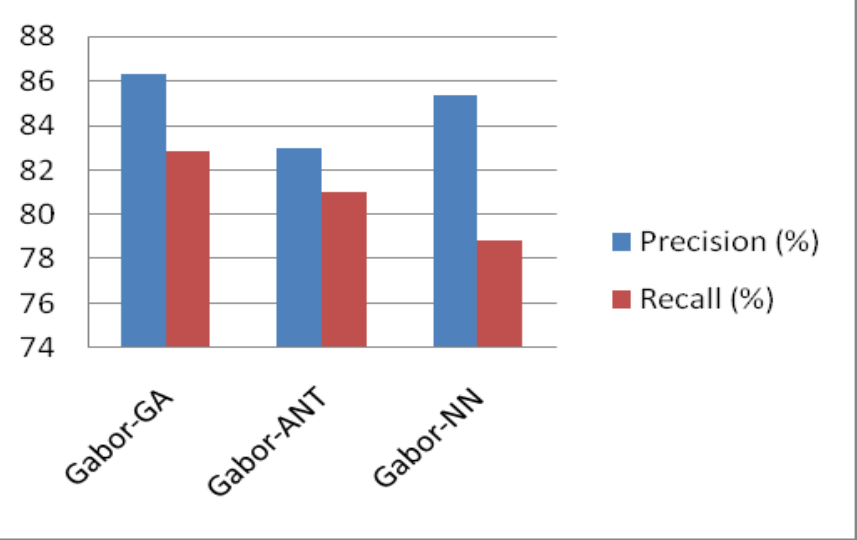

Fig5: Comparative result analysis of data set-1 in three method such as GA,ANT and NN.

The above result shows that the NN feature optimization is better than GA and ANT. 


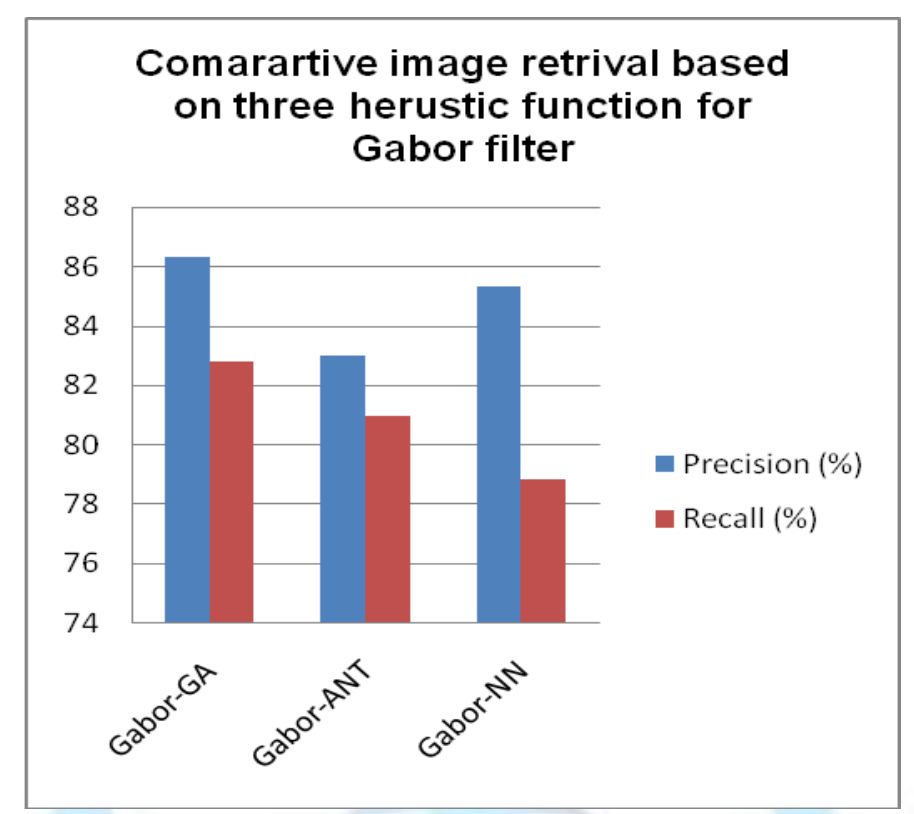

Fig 6: Comparative result analysis of data set-2 in three method such as GA,ANT and NN.

The above result shows that the GA feature optimization is better than NN and ANT.

\section{CONCLUSION AND FUTURE WORK}

In this paper we discuss the empirical evaluation of image retrieval using Gabor feature extraction technique using optimization process such as genetic algorithm, ANT colony optimization and neural network. The performance of genetic algorithm and neural network varies according to the changing of data set. The process of optimization consider as single objective function. The single objective function deals only the process of query image. In the process of review found that the shape based feature extraction is critical and most promising result in compared with other feature of image such as color and texture. The training time of neural network improved the computational overhead during the retrieval of query image. In future for better feature selection used modified ANT colony system along with neural network.

\section{ACKNOWLEDGMENTS}

Pushpa T. Tandekar is thankful to Prof. Dhirendra Kumar Jha Head of Dept. Computer Science \& Engineering Malhotra Technical Research Institute, Bhopal. For his help and support for completion of this project

\section{REFERENCES}

[1] G. Castellano, A.M. Fanelli, F. Paparella, M.A. Torsello "Fuzzy Shape Clustering for Image Retrieval "Hawaii International Conference on System Sciences, IEEE 2013, pp 1423-1429.

[2] Sonal Kothari, John H Phan, Andrew N Young and May D Wang "Histological image classification using biologically interpretable shape-based features "Medical Imaging 2013, pp 1-17.

[3] Bhailal Limbasiya, Swati Patel "Content Based Image Retrieval with Log Based Relevance Feedback Using Combination of Query Expansion and Query Point movement "IJCSMC Vol. 2, Issue. 5,2013. Pp 155-161

[4] Lining Zhang, Lipo Wang, Weisi Lin "Semisupervised Biased Maximum Margin Analysis for Interactive Image Retrieval" IEEE TRANSACTIONS ON IMAGE PROCESSING, VOL. 21, 2012. Pp 2294-2308.

[5] Saurabh Agrawal, Nishchal K Verma, Prateek Tamrakar, Pradip Sircar "Content Based Color Image Classification using SVM "Eighth International Conference on Information Technology IEEE 2011. Pp 1090-1095.

[6] Serafeim Moustakidis, Giorgos Mallinis, Nikos Koutsias, John B. Theocharis "SVM-Based Fuzzy Decision Trees for Classification of High Spatial Resolution Remote Sensing Images" IEEE TRANSACTIONS ON GEOSCIENCE AND REMOTE SENSING, VOL. 50, 2012. Pp 149-170.

[7] Weiming Hu, Nianhua Xie, Li Li, Xianglin Zeng, Stephen Maybank "A Survey on Visual Content-Based Video Indexing and Retrieval "IEEE TRANSACTIONS ON SYSTEMS, MAN, AND CYBERNETICS, vol-41, 2011. Pp 797-819.

[8] Monika Jain, Dr. S.K.Singh "A Survey On: Content Based Image Retrieval Systems Using Clustering Techniques For Large Data sets" (IJMIT) Vol.3,2011. Pp 23-40.

[9] B.Ramamurthy, K.R.Chandran "CBMIR: SHAPE-BASED IMAGE RETRIEVAL USING CANNY EDGE DETECTION AND K-MEANS CLUSTERING ALGORITHMS FOR MEDICAL IMAGES “IJEST vol 3, 2011. Pp 1870-1878. 
[10] Yi Yang, Feiping Nie, Dong Xu, Jiebo Luo "A Multimedia Retrieval Framework Based on Semi-Supervised Ranking and Relevance Feedback" IEEE TRANSACTIONS ON PATTERN ANALYSIS AND MACHINE INTELLIGENCE, VOL. 34, 2012. Pp 723-742.

[11] Yue Gao, Meng Wang, Dacheng Tao "3-D Object Retrieval and Recognition With Hypergraph Analysis "IEEE TRANSACTIONS ON IMAGE PROCESSING, VOL. 21, 2012. Pp 4290-4302.

[12] T. Karthikeyan, R. Balakrishnan, U.Karthick Kumar "Priority Based Pheromone Algorithm for Image Cluster" IEEE, 2012. Pp 560-568.

[13] Komal Asrani , Renu Jain " Comparing the impact of Clustering with Content Based Image Retrieval Approaches for Plant Identification "IJCT vol-9, 2013. Pp 985-995.

[14] Peihong Zhu, Suyash P. Awate, Samuel Gerber, Ross Whitaker "Fast Shape-Based Nearest-Neighbor Search for Brain MRIs Using Hierarchical Feature Matching" MICCAI springer, 2011. Pp 484-491.

[15] I. Samuel Peter James “ Face Image Retrieval with HSV Color Space using Clustering Techniques" The SIJ Transactions on Computer Science Engineering \& its Applications vol-1, 2013. Pp 17-21.

[16] Priyanka Dhasal , Shiv Shakti Shrivastava, Hitesh Gupta, Parmalik Kumar "An Optimized Feature SeLection for Image Classification Based on SVMACO "IJACR Volume-2, 2012. Pp 123-129.

[17] D. Zhou, J. Weston, A. Gretton, O. Bousquet, and B. Scho"lkopf, "Ranking on Data Manifolds," Proc. Advances in Neural Information Processing Systems, 2003.

[18]Wei-jiu Zhang, Li Mao, Wen-bo Xu "Automatic Image Classification Using the Classification Ant- Colony Algorithm" in International Conference on Environmental Science and Information Application Technology 2009.

[19] Siegel R, Ward E, Brawley O, Jemal A: Cancer statistics, 2011. CA Cancer J Clin 2011, pp 212-236.

[20] M. Kim, M. Madden, and T. A. Warner, "Forest type mapping using object-specific texture measures from multispectral IKONOS imagery: Segmentation quality and image classification issues," Photogramm. Eng. Remote Sens., vol. 75 , no. 7 , pp. 819-829, Jul. 2009.

\section{Author' biography with Photo}
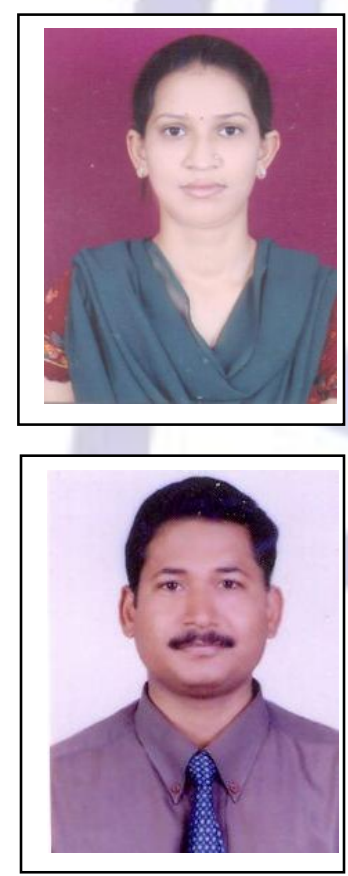

Miss.Pushpa Tejlal Tandekar is M-TECH student in Dept. of Computer Science and Engineering, Malhotra Technical Research Institute, Bhopal. She obtained his BE in Government college of Engineering,Chandrapur.She has published National level paper in Data Mining.
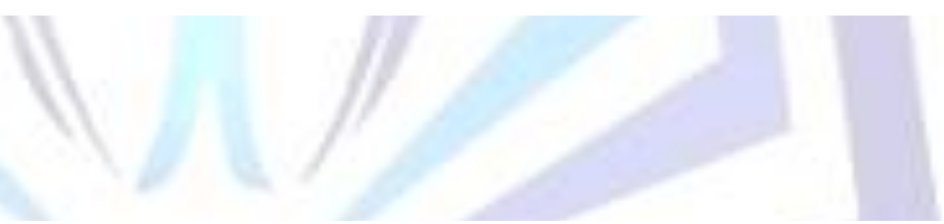

Prof. Dhirendra Kumar Jha is professor and Head of Department Computer Science \& Engineering. He has over 15 Years of teaching and research experience in Computer Science. He has published number of research paper in journals, both national and international. He obtained his BE and ME in Bhopal University and Ph.D. Persuing in, Bhopal. He has been innovated in teaching and research in area of Fuzzy logic and Fuzzy set,Embedded system and Image processing. 